\title{
Exponentially Fitted Numerical Method for Singularly Perturbed Differential-Difference Equations
}

\author{
Habtamu Garoma Debela ${ }^{D}$, Solomon Bati Kejela, and Ayana Deressa Negassa \\ Department of Mathematics, College of Natural Sciences, Jimma University, Jimma, Ethiopia \\ Correspondence should be addressed to Habtamu Garoma Debela; habte200@gmail.com
}

Received 8 April 2020; Revised 14 May 2020; Accepted 28 May 2020; Published 17 June 2020

Academic Editor: Patricia J. Y. Wong

Copyright (C) 2020 Habtamu Garoma Debela et al. This is an open access article distributed under the Creative Commons Attribution License, which permits unrestricted use, distribution, and reproduction in any medium, provided the original work is properly cited.

\begin{abstract}
This paper presents a numerical method to solve singularly perturbed differential-difference equations. The solution of this problem exhibits layer or oscillatory behavior depending on the sign of the sum of the coefficients in reaction terms. A fourthorder exponentially fitted numerical scheme on uniform mesh is developed. The stability and convergence of the proposed method have been established. The effect of delay parameter (small shift) on the boundary layer(s) has also been analyzed and depicted in graphs. The applicability of the proposed scheme is validated by implementing it on four model examples. Maximum absolute errors in comparison with the other numerical experiments are tabulated to illustrate the proposed method.
\end{abstract}

\section{Introduction}

Differential equations with a small parameter $\varepsilon(0<\varepsilon \leq 1)$ multiplying the highest order derivative are called singularly perturbed differential equations. Mathematically, any ordinary differential equation in which the highest derivative is multiplied by a small positive parameter and containing at least one delay/advance parameter is known as a singularly perturbed differential-difference equation [1]. Such type of equations arises frequently from the mathematical modeling of various practical phenomena, for example, in the modeling of the human pupil-light reflex [2], the study of bistable devices [3], and vibrational problems in control theory [4]. When perturbation parameter $\varepsilon$ is very small, most numerical methods for solving such problems may be unstable and give inaccurate results. So, it is important to develop suitable numerical methods to solve singularly perturbed delay differential equations.

Hence, in the recent times, many researchers have been trying to develop numerical methods for solving singularly perturbed delay differential equations. For example, the authors in [5] proposed a computational method of first order for singularly perturbed delay reaction-diffusion equations with layer or oscillatory behavior. The authors in [6] presented a fourth-order finite difference scheme for second-order singularly perturbed differential-difference equations with negative shift. The authors in [7] presented exponentially fitted a second-order finite difference scheme for a class of singularly perturbed delay differential equations with large delay. In [8], the numerical solution of singularly perturbed differential-difference equations with dual layer was presented. Recently, the authors in [9] presented a computational method for solving a singularly perturbed delay differential equation with twin layers or oscillatory behavior. But, still there is a lack of accuracy because the treatment of singularly perturbed problems is not trivial and the solution depends on perturbation parameter $\varepsilon$ and mesh size $h$ [10-12]. Due to this, numerical treatment of singularly perturbed delay differential equations needs improvement. Therefore, it is important to develop a more accurate and convergent numerical method for solving singularly perturbed delay differential equations.

Consider the following singularly perturbed delay differential equation of the form:

$$
\varepsilon y^{\prime \prime}+a(x) y(x-\delta)+b(x) y(x)=f(x), \quad 0<x<1,
$$

subject to the interval and boundary conditions 


$$
y(x)=\phi(x), \text { for }-\delta \leq x \leq 0 \text { and } y(1)=\varphi,
$$

where $\varepsilon$ is the perturbation parameter, $0<\varepsilon \ll 1$, and $\delta$ is a small delay parameter of $O(\varepsilon), 0<\delta \ll 1$. Also, $a(x), b(x), f(x)$, and $\phi(x)$ are bounded smooth functions and $\varphi$ is a given constant. The layer or oscillatory behavior of the problem under consideration is maintained for $\delta \neq 0$ but sufficiently small, depending on the sign of $a(x)+b(x)$, for all $x \in(0,1)$. If $a(x)+b(x)<0$, the solution of the problem in equations (1) and (2) exhibits layer behavior, and if $a(x)+$ $b(x)<0$, it exhibits oscillatory behavior. Therefore, if the solution exhibits layer behavior, there will be two boundary layers which will occur at both end points $x=0$ and $x=1[12]$.

Thus, the purpose of this study is to develop stable, convergent, and more accurate numerical method for solving singularly perturbed delay differential equations.

\section{Description of the Method}

By using Taylor series expansion in the neighborhood of $x$, we have

$$
y(x-\delta) \approx y(x)-\delta y^{\prime}(x)+O\left(\delta^{2}\right)
$$

Substituting equation (3) into equation (1), we obtain an asymptotically equivalent singularly perturbed two-point boundary value problem:

$$
\varepsilon y^{\prime \prime}(x)+p(x) y^{\prime}(x)+q(x) y(x)=f(x),
$$

where $\quad p(x)=-\delta a(x)$ and $q(x)=a(x)+b(x) \quad$ under boundary conditions

$$
y(0)=\phi_{0}, y(1)=\varphi .
$$

Discretizing the given interval $[0,1]$ in to $N$ equal parts with constant mesh size $h$, we have $x_{i}=x_{0}+i h, i=0,1,2, \ldots, N$.

Using Taylor's expansions of $y_{i+1}$ and $y_{i-1}$ up to $O\left(h^{5}\right)$, we get the finite difference approximations for $y_{i}^{\prime}$ and $y_{i}^{\prime \prime}$ as

$$
y_{i}^{\prime}=\frac{y_{i+1}-y_{i-1}}{2 h}-\frac{h^{2}}{6} y_{i}^{\prime \prime}+\tau_{1},
$$

where $\tau_{1}=-\left(h^{4} / 20\right) y^{(5)}\left(\xi_{1}\right)$, for $\xi_{1} \in\left[x_{i-1}, x_{i}\right]$, and

$$
y_{i}^{\prime \prime}=\frac{y_{i+1}-2 y_{i}+y_{i-1}}{h^{2}}-\frac{h^{2}}{12} y_{i}^{(4)}+\tau_{2} \text {, }
$$

where $\tau_{2}=-\left(h^{4} / 360\right) y^{(6)}\left(\xi_{2}\right)$, for $\xi_{2} \in\left[x_{i-1}, x_{i}\right]$.

Substituting equations (6) and (7) into equation (4) and simplifying, we obtain

$$
\frac{\varepsilon}{h^{2}}\left(y_{i+1}-2 y_{i}+y_{i-1}\right)+\frac{p_{i}}{2 h}\left(y_{i+1}-y_{i-1}\right)-\frac{h^{2}}{6} p_{i} y_{i}-\frac{\varepsilon h^{2}}{12} y_{i}^{(4)}+q_{i} y_{i}=f_{i}+\tau_{i}
$$

where $\tau_{i}=h^{4}\left(\left(p_{i} / 120\right) y^{(5)}\left(\xi_{1}\right)+(\varepsilon / 360) y^{(6)}\left(\xi_{2}\right)\right)+O\left(h^{5}\right)$ is the local truncation error and $p\left(x_{i}\right)=p_{i}, q\left(x_{i}\right)=q_{i}$, $f\left(x_{i}\right)=f_{i}$, and $y_{i} \approx y\left(x_{i}\right)$.
By successively differentiating both sides of equation (4) and evaluating at $x_{i}$ and using into equation (8), we obtain

$$
\frac{\varepsilon}{h^{2}}\left(y_{i+1}-2 y_{i}+y_{i-1}\right)+\frac{p_{i}}{2 h}\left(y_{i+1}-y_{i-1}\right)+\varepsilon\left\{\frac{h^{2} p_{i}^{2}}{6 \varepsilon}+\frac{h^{2}}{6}\left(2 p_{i}^{\prime}+q_{i}-\frac{p_{i}^{2}}{\varepsilon}\right)\right\} y_{i}^{\prime \prime}+A_{i} y_{i}^{\prime}+\left(B_{i}+b_{i}\right) y_{i}=f_{i}+C_{i}
$$

where

$$
\left\{\begin{array}{l}
A_{i}=\frac{h^{2}}{6 \varepsilon} p_{i}\left(p_{i}^{\prime}+q_{i}\right)-\frac{h^{2}}{12}\left(\frac{p_{i}}{\varepsilon}\left(p_{i}^{\prime}+q_{i}\right)-p_{i}^{\prime \prime}-2 q_{i}^{\prime}\right) \\
B_{i}=\frac{h^{2}}{6 \varepsilon} p_{i} q_{i}^{\prime}-\frac{h^{2}}{12}\left(\frac{p_{i} q_{i}^{\prime}}{\varepsilon}-q_{i}^{\prime \prime}\right), \\
C_{i}=f_{i}+\frac{a_{i} h^{2}}{12 \varepsilon} f_{i}^{\prime}+\frac{h^{2}}{12} f_{i}^{\prime \prime}+\tau_{i} .
\end{array}\right.
$$

Now introducing a fitting parameter $\sigma$ and using central difference approximation for $y_{i}^{\prime \prime}$ and $y_{i}^{\prime}$ in equation (9), we have 


$$
\frac{\sigma \varepsilon}{h^{2}}\left\{1+\frac{h^{2} p_{i}^{2}}{12 \varepsilon}+\frac{h^{2}}{12}\left(2 p_{i}^{\prime}+q_{i}\right)\right\}\left(y_{i+1}-2 y_{i}+y_{i-1}\right)+\frac{1}{2 h}\left(p_{i}+A_{i}\right)\left(y_{i+1}-y_{i-1}\right)+\left(B_{i}+q_{i}\right) y_{i}=f_{i}+C_{i}
$$

Multiplying both sides of equation (11) by $h$ and taking the limit as $h \longrightarrow 0$, we obtain

$$
\frac{\sigma}{12 \rho}\left(12+\rho^{2} p_{i}^{2}\right) \lim _{h \longrightarrow 0}\left\{y_{i+1}-2 y_{i}+y_{i-1}\right\}+\frac{p_{i}}{2} \lim _{h \longrightarrow 0}\left\{y_{i+1}-y_{i-1}\right\}=0
$$

where $\rho=h / \varepsilon$.

From the theory of singular perturbations and [13], we

Case 1. For $p(x)<0$ (right-end boundary layer), we have have two cases for $p(x)>0$ and $p(x)<0$ :

$$
\left\{\begin{array}{l}
\lim _{h \longrightarrow 0}\left(y_{i+1}-2 y_{i}+y_{i-1}\right)=\left(\phi_{0}-y_{0}(0)\right) e^{-p(0)((-1 / \varepsilon)+i \rho)}\left(e^{-p(0) \rho}+e^{p(0) \rho}-2\right) \\
\lim _{h \longrightarrow 0}\left(y_{i+1}-y_{i-1}\right)=\left(\phi_{0}-y_{0}(0)\right) e^{-p(0)((-1 / \varepsilon)+i \rho)}\left(e^{-p(0) \rho}-e^{p(0) \rho}\right)
\end{array}\right.
$$

Thus, from equation (12), we get

$$
\sigma(0)=\frac{6 \rho p(0)}{\left(12+\rho^{2} p^{2}(0)\right)} \operatorname{coth}\left(\frac{p(0) \rho}{2}\right) .
$$

$$
\left\{\begin{array}{l}
\lim _{h \longrightarrow 0}\left(y_{i+1}-2 y_{i}+y_{i-1}\right)=\left(\varphi_{0}-y_{0}(1)\right) e^{-p(1)(-1 / \varepsilon+i \rho)}\left(e^{-p(1) \rho}+e^{p(1) \rho}-2\right), \\
\lim _{h \longrightarrow 0}\left(y_{i+1}-y_{i-1}\right)=\left(\varphi_{0}-y_{0}(1)\right) e^{-p(1)(-1 / \varepsilon+i \rho)}\left(e^{-p(1) \rho}-e^{p(1) \rho}\right) .
\end{array}\right.
$$

Thus, from equation (12), we get

$$
\sigma(1)=\frac{6 \rho p(1)}{\left(12+\rho^{2} p^{2}(1)\right)} \operatorname{coth}\left(\frac{p(1) \rho}{2}\right) .
$$

In general, for discretization, we take a variable fitting parameter as

$$
\sigma_{i}=\frac{6 \rho_{i} p_{i}}{\left(12+\left(\rho_{i} p_{i}\right)^{2}\right)} \operatorname{coth}\left(\frac{p_{i} \rho_{i}}{2}\right)
$$

where $\rho_{i}=h / \varepsilon$.

Simplifying equation (11), we get the tridiagonal system of the equation as follows:

$$
L^{N} \equiv E_{i} y_{i-1}-F_{i} y_{i}+G_{i} y_{i+1}=H_{i}, \quad \text { for } i=1,2, \ldots, N-1,
$$

where

$$
\left\{\begin{array}{l}
E_{i}=\frac{\sigma_{i} \varepsilon}{h^{2}}+\frac{\sigma_{i} p_{i}^{2}}{12}+\frac{\sigma_{i} \varepsilon}{12}\left(2 p_{i}^{\prime}+q_{i}\right)-\frac{1}{2 h}\left(p_{i}+A_{i}\right), \\
F_{i}=\frac{2 \sigma_{i} \varepsilon}{h^{2}}+\frac{\sigma_{i} p_{i}^{2}}{6}+\frac{\sigma_{i} \varepsilon}{6}\left(2 p_{i}^{\prime}+q_{i}\right)-p_{i}-B_{i}, \\
G_{i}=\frac{\sigma_{i} \varepsilon}{h^{2}}+\frac{\sigma_{i} p_{i}^{2}}{12}+\frac{\sigma_{i} \varepsilon}{12}\left(2 p_{i}^{\prime}+q_{i}\right)+\frac{1}{2 h}\left(p_{i}+A_{i}\right), \\
H_{i}=f_{i}+C_{i} .
\end{array}\right.
$$

The tridiagonal system in equation (18) can be easily solved by the Thomas algorithm with help of Matlab 2013.

\section{Stability and Convergence Analysis}

Case 1: Layer behavior (i.e., $a(x)+b(x)<0$, for $x \in(0,1)$; thus, $q(x)<0$, since $\varepsilon>0$ ). 
Lemma 1. If $y(0) \geq 0$ and $L y(x) \leq 0$, for all $x \in(0,1)$, then the solution $y(x) \geq 0$ for all $x \in(0,1)$ for equations (4) and (5).

Proof. Suppose $t \in(0,1)$, such that $y(t)=\min _{x \in(0,1)} y(x)$ and $y(t)<0$. Since $t \notin\{0,1\}$ and is a point of minima, then $y(t)=0$ and $y^{\prime \prime}(t) \geq 0$.

Therefore, we have $L y(t)=\varepsilon y^{\prime \prime}(t)+p(t) y^{\prime}(t)+$ $q(t) y(t)>0$, since $y(t)<0$ (by assumption) and $q(t)<0$. But this is a contradiction. Then, it follows that $y(x) \geq 0$ for $x \in(0,1)$.

Theorem 1. If the solution of the problem in equations (4) and (5) satisfies

$$
|y(x)| \leq K \max \left\{|y(0)|, \max _{x \in(0,1)}|L y(x)|\right\},
$$

for some constant $K \geq 1$, then the solution is stable.

Proof. We define two functions: $\psi^{ \pm}(x)=K \max \{|y(0)|$, $\left.\max _{x \in(0,1)}|L y(x)|\right\} \pm y(x)$. Then, we get $\psi^{ \pm}(0) \geq 0$ and
$L \psi^{ \pm}(x)=K q(x) \max \left\{|y(0)|, \max _{x \in(0,1)}|L y(x)|\right\} \pm L y(x) \leq 0$,

since $q(x)<0$ and for suitable choice of $K$. Therefore, by Lemma 1 , we get $\psi^{ \pm}(x) \geq 0$, for all $x \in(0,1)$. So, $|y(x)| \leq K \max \left\{|y(0)|, \max _{x \in(0,1)}|L y(x)|\right\}$.

Hence, the stability of the solutions of the problem in equations (4) and (5) is proved for the case of the layer behavior.

Lemma 2. The finite difference operator $L^{N}$ in equation (13) satisfies the discrete minimum principle; i.e., if $w_{i}$ is any mesh function such that $w_{0} \geq 0$ and $L^{N} w_{i} \leq 0$, for all $x_{i} \in(0,1)$, then $w_{i} \geq 0$ for all $x_{i} \in(0,1)$.

Proof. Suppose there exists a positive integer $k$ such that $w_{k}<0$ and $w_{i}=\min _{0 \leq i \leq N} w_{i}$. Then, from equation (13), we have

$$
\begin{aligned}
L^{N} w_{k}= & E_{k} w_{k-1}-F_{k} w_{k}+G_{k} w_{k+1} \\
= & \left\{\frac{\sigma_{k} \varepsilon}{h^{2}}+\frac{\sigma_{k} p_{k}^{2} 1}{12}+\frac{\sigma_{k} \varepsilon}{12}\left(2 p_{k}^{\prime}+q_{k}\right)\right\}\left(w_{k+1}-2 w_{k}+w_{k-1}\right)+\frac{1}{2 h}\left(p_{k}+A_{k}\right)\left(w_{k+1}-w_{k-1}\right)+\left(p_{k}+B_{k}\right) w_{k} \\
= & \left\{\frac{\sigma_{k} \varepsilon}{h^{2}}+\frac{\sigma_{k} p_{k}^{2} 1}{12}+\frac{\sigma_{k} \varepsilon}{12}\left(2 p_{k}^{\prime}+q_{k}\right)\right\}\left(w_{i+1}-w_{k}\right)+\left\{\frac{\sigma_{k} \varepsilon}{h^{2}}+\frac{\sigma_{k} p_{k}^{2} 1}{12}+\frac{\sigma_{k} \varepsilon}{12}\left(2 p_{k}^{\prime}+q_{k}\right)\right\}\left(w_{k-1}-w_{k}\right) \\
& +\frac{1}{2 h}\left(p_{k}+\left(\frac{h^{2}}{6 \varepsilon} p_{k}\left(p_{k}^{\prime}+q_{k}\right)-\frac{h^{2}}{12}\left(\frac{p_{k}}{\varepsilon}\left(p_{k}^{\prime}+q_{k}\right)-p_{k}^{\prime \prime}-2 q_{k}^{\prime}\right)\right)\right)\left(w_{i+1}-w_{i-1}\right) \\
& +\left(p_{k}+\left(\frac{h^{2}}{6 \varepsilon} p_{i} q_{i}^{\prime}-\frac{h^{2}}{12}\left(\frac{p_{i} q_{i}^{\prime}}{\varepsilon}-q_{k}^{\prime \prime}\right)\right)\right) w_{k} .
\end{aligned}
$$

For sufficiently small $h$ and for suitable value of $p_{k}$, we obtain $L^{N} w_{k}>0$. However, $w_{k}<0$ (by the assumption) and $B_{k} \longrightarrow p_{k}<0$. But, this is a contradiction. Hence, $w_{i} \geq 0$ for all $x_{i} \in(0,1)$.

Theorem 2. The finite difference operator $L^{N}$ in equation (13) is stable for $a(x)+b(x)<0$, if $w_{i}$ is any mesh function, then $\left|w_{i}\right| \leq K \max \left\{\left|w_{0}\right|, \max _{x_{i} \in(0,1)}\left|L w_{i}\right|\right\}$, for some constant $K \geq 1$.

Proof. We define two functions:

$$
\psi^{ \pm}=K \max \left\{|w(0)|, \max _{x \in(0,1)}\left|L w_{i}\right|\right\} \pm w_{i} .
$$

Then, similar to Theorem 1 , we get $\psi^{ \pm}(0) \geq 0$ and

$$
L \psi^{ \pm}=K q_{i} \max \left\{\left|w_{0}\right|, \max _{x \in(0,1)}\left|L w_{i}\right|\right\} \pm L w_{i} \leq 0,
$$

since $a_{i}+b_{i}<0 \Rightarrow q(x)<0$ and $K \geq 1$, therefore, by Lemma 2 , we get $\psi_{i}^{ \pm} \geq 0$, for all $x_{i} \in(0,1)$. $\Rightarrow \psi_{i}^{ \pm} \equiv C \max \left\{\left|w_{0}\right|, \max _{x_{i} \in(0,1)}\left|L w_{i}\right|\right\} \pm w_{i} \geq 0$.

Thus, $\left|w_{i}\right| \leq C \max \left\{\left|w_{0}\right|, \max _{x_{i} \in(0,1)}\left|L w_{i}\right|\right\}$.

This proves the stability of the scheme for the case of layer behavior.

Case 2: oscillatory behavior (i.e., $a(x)+b(x)>0$, for $x \in(0,1)$; thus, $q(x)>0$, since $\varepsilon>0)$.

The continuous maximum principle and stability of the solution of equations (4) and (5) are presented as follows for the case of oscillatory behavior.

Lemma 3. If $y(0) \geq 0$ and $L y(x) \geq 0$, for all $x \in(0,1)$, then the solution $y(x) \geq 0$ for all $x \in(0,1)$ for equations (4) and (5). 
Proof. Suppose $t \in(0,1)$, such that $y(t)=\max _{x \in(0,1)} y(x)$ and $y(t)<0$. Since $t \notin\{0,1\}$ and is a point of maxima, therefore, $y^{\prime}(t)=0$ and $y^{\prime \prime}(t) \leq 0$. Therefore, we have $L y(t)=\varepsilon y^{\prime \prime}(t)+p(t) y^{\prime}(t)+q(t) y(t)<0$, since $y(t)<0$ (by assumption) and $q(t)<0$. But this is a contradiction. Hence, $y(x) \geq 0$ for $x \in(0,1)$.

Theorem 3. If the solution of the problem in equations (4) and (5) satisfies

$$
|y(x)| \leq K \max \left\{|y(0)|, \max _{x \in(0,1)}|L y(x)|\right\},
$$

for some constant, $k \geq 1$.
Proof. The proof is analogous to Theorem 1. Hence, the stability of the solutions of the problem in equations (4) and (5) is proved for the case of oscillatory behavior. Now, we present the stability of the discrete problem in equation (13) for the case of oscillatory behavior.

Lemma 4. The finite difference operator $L^{N}$ in equation (13) satisfies the discrete maximum principle, if $w_{i}$ is any mesh function such that $w_{0} \geq 0$ and $L^{N} w_{i} \geq 0$, for all $x_{i} \in(0,1)$, then $w_{i} \geq 0$ for all $x_{i} \in(0,1)$.

Proof. Suppose there exists a positive integer $k$ such that $w_{k}<0$ and $w_{i}=\max _{0 \leq i \leq N} w_{i}$. Then, from equation (13), we have

$$
\begin{aligned}
L^{N} w_{k}= & E_{k} w_{k-1}-F_{k} w_{k}+G_{k} w_{k+1} \\
= & \left\{\frac{\sigma_{k} \varepsilon}{h^{2}}+\frac{\sigma_{k} p_{k}^{2} 1}{12}+\frac{\sigma_{k} \varepsilon}{12}\left(2 p_{k}^{\prime}+q_{k}\right)\right\}\left(w_{k+1}-2 w_{k}+w_{k-1}\right)+\frac{1}{2 h}\left(p_{k}+A_{k}\right)\left(w_{k+1}-w_{k-1}\right)+\left(p_{k}+B_{k}\right) w_{k} \\
= & \left\{\frac{\sigma_{k} \varepsilon}{h^{2}}+\frac{\sigma_{k} p_{k}^{2} 1}{12}+\frac{\sigma_{k} \varepsilon}{12}\left(2 p_{k}^{\prime}+q_{k}\right)\right\}\left(w_{i+1}-w_{k}\right)+\left\{\frac{\sigma_{k} \varepsilon}{h^{2}}+\frac{\sigma_{k} p_{k}^{2} 1}{12}+\frac{\sigma_{k} \varepsilon}{12}\left(2 p_{k}^{\prime}+q_{k}\right)\right\}\left(w_{k-1}-w_{k}\right) \\
& +\frac{1}{2 h}\left(p_{k}+\left(\frac{h^{2}}{6 \varepsilon} p_{k}\left(p_{k}^{\prime}+q_{k}\right)-\frac{h^{2}}{12}\left(\frac{p_{k}}{\varepsilon}\left(p_{k}^{\prime}+q_{k}\right)-p_{k}^{\prime \prime}-2 q_{k}^{\prime}\right)\right)\right)\left(w_{i+1}-w_{i-1}\right) \\
& +\left(p_{k}+\left(\frac{h^{2}}{6 \varepsilon} p_{i} q_{k}^{\prime}-\frac{h^{2}}{12}\left(\frac{p_{i} q_{i}^{\prime}}{\varepsilon}-q_{k}^{\prime \prime}\right)\right)\right) w_{k} .
\end{aligned}
$$

For sufficiently small $h$ and for suitable value of $p_{k}$, we obtain $L^{N} w_{k}<0$. However, $w_{k}<0$ (by the assumption) and $B_{k} \longrightarrow p_{k}>0$. But, this is a contradiction. Hence, $w_{i} \geq 0$ for all $x_{i} \in(0,1)$.

Theorem 4. The finite difference operator $L^{N}$ in equation (13) is stable for $a(x)+b(x)>0$, (i.e. $q(x)>0$ ), if $w_{i}$ is any mesh function, then $\left|w_{i}\right| \leq K \max \left\{\left|w_{0}\right|, \max _{x_{i} \in(0,1)}\left|L w_{i}\right|\right\}$, for some constant $K \geq 1$.

Proof. The proof is similar to Theorem 2. This proves the scheme for the case of oscillatory behavior.
Definition 1. (uniform convergence). Let $y$ be a solution of equations (1) and (2). Consider a difference scheme for solving equations (1) and (2). If the scheme has a numerical solution $y^{N}$ that satisfies $\left\|y-y^{N}\right\| \leq C h^{p}$, where $C>0$ and $p>0$ are independent of $\varepsilon$ and of mesh size $h$, then we say the scheme uniformly convergent to $y$ with respect to the norm $\|\cdot\|[14]$.

Lemma 5. The bound for derivative of the solution $y(x)$ of the problem (1)-(3) when $x \in \Omega_{1}=(0,1)$ is given by

$$
\begin{cases}\left|y^{(k)}(x)\right| \leq C\left(1+\varepsilon^{-k} \exp \left(\frac{-\alpha x_{j}}{\varepsilon}\right)\right), & 0 \leq k \leq 4, j=1,2, \ldots, N-1, \\ \left|y^{(k)}(x)\right| \leq C\left(1+\varepsilon^{-k} \exp \left(\frac{-\alpha\left(1-x_{j}\right)}{\varepsilon}\right)\right), & 0 \leq k \leq 4, j=1,2, \ldots, N-1 .\end{cases}
$$


Proof. For the proof, refer [15]

For any mesh function $z_{i}$, define the following difference operators:

$$
\begin{aligned}
D^{+} z_{i} & =\frac{z_{i+1}-z_{i}}{h}, \\
D^{-} z_{i} & =\frac{z_{i}-z_{i-1}}{h}, \\
D^{0} z_{i} & =\frac{z_{i+1}-z_{i-1}}{2 h}, \\
D^{+} D^{-} z_{i} & =\frac{z_{i+1}-2 z_{i}+z_{i-1}}{h^{2}} .
\end{aligned}
$$

$$
L^{N}\left(y\left(x_{i}\right)-y_{i}\right)=\varepsilon \sigma(\rho)\left(\frac{\mathrm{d}^{2}}{\mathrm{~d} x^{2}}-D^{+} D^{-}+\frac{h^{2}}{12} \frac{\mathrm{d}^{4}}{\mathrm{~d} x^{4}}\right) y\left(x_{i}\right)+p\left(x_{i}\right)\left(\frac{\mathrm{d}}{\mathrm{d} x}-D^{0}+\frac{h^{2}}{6} \frac{\mathrm{d}^{2}}{\mathrm{~d} x^{2}}\right) y\left(x_{i}\right),
$$

where

$$
\varepsilon \sigma(\rho)=\varepsilon\left(\frac{6 \rho p(0)}{\left(12+\rho^{2} p^{2}(0)\right)} \operatorname{coth}\left(\frac{p(0) \rho}{2}\right)\right) \approx p(0) \frac{N^{-1}}{2} \operatorname{coth}\left(p(0) \frac{N^{-1}}{2 \varepsilon}\right)
$$

since $\rho=N^{-1} / \varepsilon$. In our assumption, $\varepsilon \leq h=N^{-1}$.

By considering $N$ is fixed and taking the limit for $\varepsilon \longrightarrow 0$, we obtain the following:

$$
\lim _{\varepsilon \longrightarrow 0} \varepsilon \sigma(\rho)=\lim _{\varepsilon \longrightarrow 0} p(0) \frac{N^{-1}}{2} \operatorname{coth}\left(p(0) \frac{N^{-1}}{2 \varepsilon}\right)=C N^{-1} .
$$

where

$$
\left\|\frac{\mathrm{d}^{k}\left(y\left(x_{i}\right)\right)}{\mathrm{d} x^{k}}\right\|=\sup _{x_{i} \in\left(x_{0}, x_{N}\right)}\left(\frac{\mathrm{d}^{k} y\left(x_{i}\right)}{\mathrm{d} x^{k}}\right), \quad k=3,4 .
$$

Now using the bounds and the assumption $\varepsilon \leq N^{-1}$, equation (30) reduces to

From Taylor series expansion, the bound for the difference becomes

$$
\left\{\begin{array}{l}
\left\|\left(\frac{\mathrm{d}^{2}}{\mathrm{~d} x^{2}}-D^{+} D^{-}\right) y\left(x_{i}\right)\right\| \leq C N^{-3}\left\|\frac{\mathrm{d}^{4}\left(y\left(x_{i}\right)\right)}{\mathrm{d} x^{4}}\right\|, \\
\left\|\left(\frac{\mathrm{d}}{\mathrm{d} x}-D^{0}\right) y\left(x_{i}\right)\right\| \leq C N^{-2}\left\|\frac{\mathrm{d}^{3}\left(y\left(x_{i}\right)\right)}{\mathrm{d} x^{3}}\right\|,
\end{array}\right.
$$

$$
\begin{aligned}
\left\|L^{N}\left(y\left(x_{i}\right)-y_{i}\right)\right\| & =\left\|\varepsilon \sigma(\rho)\left(\frac{\mathrm{d}^{2}}{\mathrm{~d} x^{2}}-D^{+} D^{-}\right) y\left(x_{i}\right)+\varepsilon \sigma(\rho) \frac{h^{2}}{12} y_{i}^{(4)}+p\left(x_{i}\right)\left(\frac{\mathrm{d}}{\mathrm{d} x}-D^{0}\right) y\left(x_{i}\right)+\frac{h^{2}}{6} y_{i}^{(2)}\right\| \\
& \leq\left\|\varepsilon \sigma(\rho)\left(\frac{\mathrm{d}^{2}}{\mathrm{~d} x^{2}}-D^{+} D^{-}\right) y\left(x_{i}\right)\right\|+\left\|\varepsilon \sigma(\rho) \frac{h^{2}}{12} y_{i}^{(4)}\right\|+\left\|p\left(x_{i}\right)\left(\frac{\mathrm{d}}{\mathrm{d} x}-D^{0}\right) y\left(x_{i}\right)\right\|+\left\|\frac{h^{2}}{6} y_{i}^{(2)}\right\| \\
& \leq C N^{-3}\left\|\frac{\mathrm{d}^{4}\left(y\left(x_{i}\right)\right)}{\mathrm{d} x^{4}}\right\|+C N^{-2}\left\|\frac{\mathrm{d}^{3}\left(y\left(x_{i}\right)\right)}{\mathrm{d} x^{3}}\right\|+C N^{-2}\left\|\frac{\mathrm{d}^{2}\left(y\left(x_{i}\right)\right)}{\mathrm{d} x^{2}}\right\| .
\end{aligned}
$$


Here, the target is to show the scheme convergence independent on the number of mesh points.
By using the bounds for the derivatives of the right layer solution in Lemma 5 , we obtain

$$
\begin{aligned}
\left\|L^{N}\left(y\left(x_{i}\right)-y_{i}\right)\right\| \leq & C N^{-3}\left\|\frac{\mathrm{d}^{4}\left(y\left(x_{i}\right)\right)}{\mathrm{d} x^{4}}\right\|+C N^{-2}\left\|\frac{\mathrm{d}^{3}\left(y\left(x_{i}\right)\right)}{\mathrm{d} x^{3}}\right\|+C N^{-2}\left\|\frac{\mathrm{d}^{2}\left(y\left(x_{i}\right)\right)}{\mathrm{d} x^{2}}\right\| \\
\leq & C N^{-3}\left(1+\varepsilon^{-4} \exp \left(\frac{-\alpha\left(1-x_{j}\right)}{\varepsilon}\right)\right)+C N^{-2}\left(1+\varepsilon^{-3} \exp \left(\frac{-\alpha\left(1-x_{j}\right)}{\varepsilon}\right)\right) \\
& +C N^{-2}\left(1+\varepsilon^{-2} \exp \left(\frac{-\alpha\left(1-x_{j}\right)}{\varepsilon}\right)\right) \\
\leq & C N^{-4}\left(1+\varepsilon^{-4} \exp \left(\frac{-\alpha\left(1-x_{j}\right)}{\varepsilon}\right)\right), \text { since } \varepsilon^{-4} \geq \varepsilon^{-3} \geq \varepsilon^{-2} .
\end{aligned}
$$

Lemma 6. For a fixed mesh and for $\varepsilon \longrightarrow 0$, it holds

$$
\lim _{\varepsilon \longrightarrow 0} \max _{1 \leq j \leq N-1} \frac{\exp \left(-\alpha\left(1-x_{j}\right) / \varepsilon\right)}{\varepsilon^{m}}=0, \quad m=1,2,3, \ldots
$$

Proof. Refer from [16].

By using Lemma 6 into equation (36), it results in

$$
\left\|L^{h}\left(y\left(x_{i}\right)-y_{i}\right)\right\| \leq C N^{-4} \text {. }
$$

Hence, by discrete maximum principle, we obtain

$$
\left\|y\left(x_{i}\right)-y_{i}\right\| \leq C N^{-4} .
$$

Thus, result of equation (39) shows equation (29). Hence proved.

Remark 1. A similar analysis for convergence may be carried out for the left layer case.

\section{Numerical Examples and Results}

To demonstrate the applicability of the method, we implemented the method on four numerical examples, two with twin boundary layers and two with oscillatory behavior. Since those examples have no exact solution, the numerical solutions are computed using the double mesh principle. The maximum absolute errors are computed using the double mesh principle given by

$$
Z_{h}=\max _{i}\left|y_{i}^{h}-y_{i}^{h / 2}\right|, \quad i=1,2, \ldots, N-1,
$$

where $y_{i}^{h}$ is the numerical solution on the mesh $\left\{x_{i}\right\}_{1}^{N}$ at the nodal point $x_{i}$ and $x_{i}=x_{0}+i h, i=1,2, \ldots, N-1$ and $y_{i}^{h / 2}$ is the numerical solution on a mesh, obtained by bisecting the original mesh with $N$ number of intervals [9].

Example 1. Consider the singularly perturbed delay reaction-diffusion equation with layer behavior

$$
\varepsilon y^{\prime \prime}(x)+0.25 y(x-\delta)-y(x)=1,
$$

under the interval and boundary conditions

$$
\begin{gathered}
y(x)=1, \\
-\delta \leq x \leq 0, \\
y(1)=0 .
\end{gathered}
$$

The maximum absolute errors are presented in Tables 1 and 2 for different values of $\varepsilon$ and $\delta$.

The graph of the computed solution for $\varepsilon=0.01$ and different values of $\delta$ is also given in Figure 1 .

Example 2. Consider the singularly perturbed delay reaction-diffusion equation with layer behavior

$$
\varepsilon y^{\prime \prime}(x)-2 y(x-\delta)-y(x)=1
$$

under the interval and boundary conditions

$$
\begin{gathered}
y(x)=1, \\
-\delta \leq x \leq 0, \\
y(1)=0 .
\end{gathered}
$$

The maximum absolute errors are presented in Tables 3 and 4 for different values of $\varepsilon$ and $\delta$.

The graph of the computed solution for $\varepsilon=0.01$ and different values of $\delta$ is also given in Figure 2 .

Example 3. Consider the singularly perturbed delay reaction-diffusion equation with oscillatory behavior

$$
\varepsilon y^{\prime \prime}(x)+0.25 y(x-\delta)+y(x)=1,
$$

under the interval and boundary conditions

$$
\begin{gathered}
y(x)=1, \\
-\delta \leq x \leq 0, \\
y(1)=0 .
\end{gathered}
$$

The maximum absolute errors are presented in Table 5 for different values of $\delta$. 
TABLE 1: The maximum absolute errors of Example 1 for different values of $\delta$ with $\varepsilon=0.1$.

\begin{tabular}{|c|c|c|c|c|c|}
\hline$\delta \downarrow$ & $N=100$ & $N=200$ & $N=300$ & $N=400$ & $N=500$ \\
\hline \multicolumn{6}{|c|}{ Present method } \\
\hline 0.03 & $1.1784 e-09$ & $7.3658 e-11$ & $1.4572 e-11$ & $4.9336 e-12$ & $1.9272 e-12$ \\
\hline 0.05 & $1.1796 e-09$ & $7.3727 e-11$ & $1.4279 e-11$ & $4.8657 e-12$ & $2.3070 e-12$ \\
\hline 0.09 & $1.1790 e-09$ & $7.3690 e-11$ & $1.4548 e-11$ & $4.5787 e-12$ & $2.5597 e-12$ \\
\hline \multicolumn{6}{|c|}{ Method in [17] } \\
\hline 0.03 & $2.1999 e-03$ & $1.1041 e-03$ & $7.3705 e-04$ & $5.5315 e-04$ & $4.4269 e-04$ \\
\hline 0.05 & $2.2012 e-03$ & $1.1049 e-03$ & $7.3749 e-04$ & $5.5345 e-04$ & $4.4293 e-04$ \\
\hline 0.09 & $2.1999 e-03$ & $1.1038 e-03$ & $7.3676 e-04$ & $7.3676 e-04$ & $4.4247 e-04$ \\
\hline
\end{tabular}

TABLE 2: The maximum absolute errors of Example 1 for different values of $\varepsilon$ with $\delta=0.5 \varepsilon$.

\begin{tabular}{|c|c|c|c|c|c|}
\hline$\varepsilon$ & $N=16$ & $N=32$ & $N=64$ & $N=128$ & $N=256$ \\
\hline \multicolumn{6}{|c|}{ Present method } \\
\hline $2^{-4}$ & $4.5775 e-06$ & $2.8651 e-07$ & $1.7913 e-08$ & $1.1197 e-09$ & $6.9995 e-11$ \\
\hline $2^{-5}$ & $1.6246 e-05$ & $1.0190 e-06$ & $6.3830 e-08$ & $3.9901 e-09$ & $2.4940 e-10$ \\
\hline $2^{-6}$ & $5.9281 e-05$ & $3.7757 e-06$ & $2.3632 e-07$ & $1.4791 e-08$ & $9.2453 e-10$ \\
\hline $2^{-7}$ & $2.2949 e-04$ & $1.4731 e-05$ & $9.2549 e-07$ & $5.7989 e-08$ & $3.6250 e-09$ \\
\hline $2^{-8}$ & $9.1215 e-04$ & $5.8144 e-05$ & $3.6824 e-06$ & $2.3104 e-07$ & $1.4448 e-08$ \\
\hline $2^{-9}$ & $3.5308 e-03$ & $2.2815 e-04$ & $1.4669 e-05$ & $9.2088 e-07$ & $5.7724 e-08$ \\
\hline $2^{-10}$ & $1.1709 e-02$ & $9.1043 e-04$ & $5.8034 e-05$ & $3.6736 e-06$ & $2.3055 e-07$ \\
\hline \multicolumn{6}{|c|}{ Method in [17] } \\
\hline $2^{-4}$ & $1.8632 e-02$ & $9.6189 e-03$ & $4.8865 e-03$ & $2.4643 e-03$ & $1.2376 e-03$ \\
\hline $2^{-5}$ & $2.8161 e-02$ & $1.4818 e-02$ & $7.6255 e-03$ & $3.8713 e-03$ & $1.9509 e-03$ \\
\hline $2^{-6}$ & $3.7958 e-02$ & $2.0967 e-02$ & $1.0977 e-02$ & $5.6273 e-03$ & $2.8498 e-03$ \\
\hline $2^{-7}$ & $5.0640 e-02$ & $2.8316 e-02$ & $1.5267 e-02$ & $7.9105 e-03$ & $4.0287 e-03$ \\
\hline $2^{-8}$ & $6.3580 e-02$ & $3.7706 e-02$ & $2.0984 e-02$ & $1.1012 e-02$ & $5.6555 e-03$ \\
\hline $2^{-9}$ & $8.3843 e-02$ & $5.0477 e-02$ & $2.8297 e-02$ & $1.5261 e-02$ & $7.9111 e-03$ \\
\hline $2^{-10}$ & $9.9137 e-02$ & $6.3529 e-02$ & $3.7660 e-02$ & $2.0974 e-02$ & $1.1011 e-02$ \\
\hline
\end{tabular}

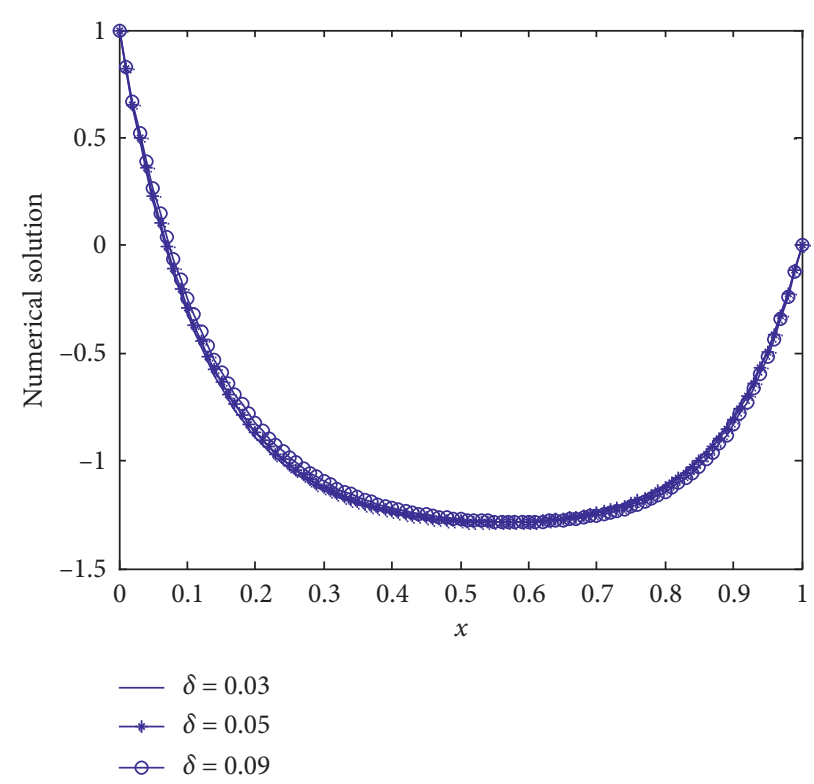

Figure 1: The numerical solution of Example 1 with $\varepsilon=0.01$ and $N=100$. 
TABLE 3: The maximum absolute errors of Example 2 for different values of $\delta$ with $\varepsilon=0.1$.

\begin{tabular}{|c|c|c|c|c|c|}
\hline$\delta \downarrow$ & $N=100$ & $N=200$ & $N=300$ & $N=400$ & $N=500$ \\
\hline \multicolumn{6}{|c|}{ Present method } \\
\hline 0.03 & $8.2805 e-09$ & $5.1758 e-10$ & $1.0224 e-10$ & $3.2348 e-11$ & $1.3243 e-11$ \\
\hline 0.05 & $7.7093 e-09$ & $4.8202 e-10$ & $9.5110 e-11$ & $3.0120 e-11$ & $1.2325 e-11$ \\
\hline 0.09 & $6.0459 e-09$ & $3.7806 e-10$ & $7.4682 e-11$ & $2.3630 e-11$ & $9.6788 e-12$ \\
\hline \multicolumn{6}{|c|}{ Method in [17] } \\
\hline 0.03 & $3.1674 e-03$ & $1.6058 e-03$ & $1.0754 e-03$ & $8.0837 e-04$ & $6.4760 e-04$ \\
\hline 0.05 & $3.1437 e-03$ & $1.5949 e-03$ & $1.0685 e-03$ & $8.0338 e-04$ & $6.4367 e-04$ \\
\hline 0.09 & $3.0784 e-03$ & $1.5660 e-03$ & $1.0502 e-03$ & $7.9000 e-04$ & $6.3310 e-04$ \\
\hline
\end{tabular}

TABLe 4: The maximum absolute errors of Example 2 for different values of $\varepsilon$ with $\delta=0.5 \varepsilon$.

\begin{tabular}{|c|c|c|c|c|c|}
\hline$\varepsilon$ & $N=16$ & $N=32$ & $N=64$ & $N=128$ & $N=256$ \\
\hline \multicolumn{6}{|c|}{ Present method } \\
\hline $2^{-4}$ & $3.0267 e-05$ & $1.9031 e-06$ & $1.1950 e-07$ & $7.4728 e-09$ & $4.6725 e-10$ \\
\hline $2^{-5}$ & $1.1987 e-04$ & $7.8382 e-06$ & $4.9134 e-07$ & $3.0732 e-08$ & $1.9223 e-09$ \\
\hline $2^{-6}$ & $4.9863 e-04$ & $3.1795 e-05$ & $1.9986 e-06$ & $1.2579 e-07$ & $7.8649 e-09$ \\
\hline $2^{-7}$ & $1.9386 e-03$ & $1.2530 e-04$ & $8.1293 e-06$ & $5.0955 e-07$ & $3.1919 e-08$ \\
\hline $2^{-8}$ & $6.4424 e-03$ & $5.1006 e-04$ & $3.2516 e-05$ & $2.0492 e-06$ & $1.2889 e-07$ \\
\hline $2^{-9}$ & $1.7543 e-02$ & $1.9764 e-03$ & $1.2772 e-04$ & $8.2523 e-06$ & $5.1725 e-07$ \\
\hline $2^{-10}$ & $3.8002 e-02$ & $6.5572 e-03$ & $5.1492 e-04$ & $3.2824 e-05$ & $2.0727 e-06$ \\
\hline \multicolumn{6}{|c|}{ Method in [17] } \\
\hline $2^{-4}$ & $2.1118 e-02$ & $1.1692 e-02$ & $6.1941 e-03$ & $3.1887 e-03$ & $1.6178 e-03$ \\
\hline $2^{-5}$ & $2.7872 e-02$ & $1.6023 e-02$ & $8.6367 e-03$ & $4.4957 e-03$ & $2.2948 e-03$ \\
\hline $2^{-6}$ & $3.5711 e-02$ & $2.1293 e-02$ & $1.1869 e-02$ & $6.2731 e-03$ & $3.2240 e-03$ \\
\hline $2^{-7}$ & $4.6679 e-02$ & $2.8350 e-02$ & $1.6107 e-02$ & $8.6728 e-03$ & $4.5120 e-03$ \\
\hline $2^{-8}$ & $5.4895 e-02$ & $3.6018 e-02$ & $2.1373 e-02$ & $1.1929 e-02$ & $6.2847 e-03$ \\
\hline $2^{-9}$ & $5.7371 e-02$ & $4.7254 e-02$ & $2.8581 e-02$ & $1.6140 e-02$ & $8.6961 e-03$ \\
\hline $2^{-10}$ & $5.7878 e-02$ & $5.5695 e-02$ & $3.6153 e-02$ & $2.1406 e-02$ & $1.1956 e-02$ \\
\hline
\end{tabular}

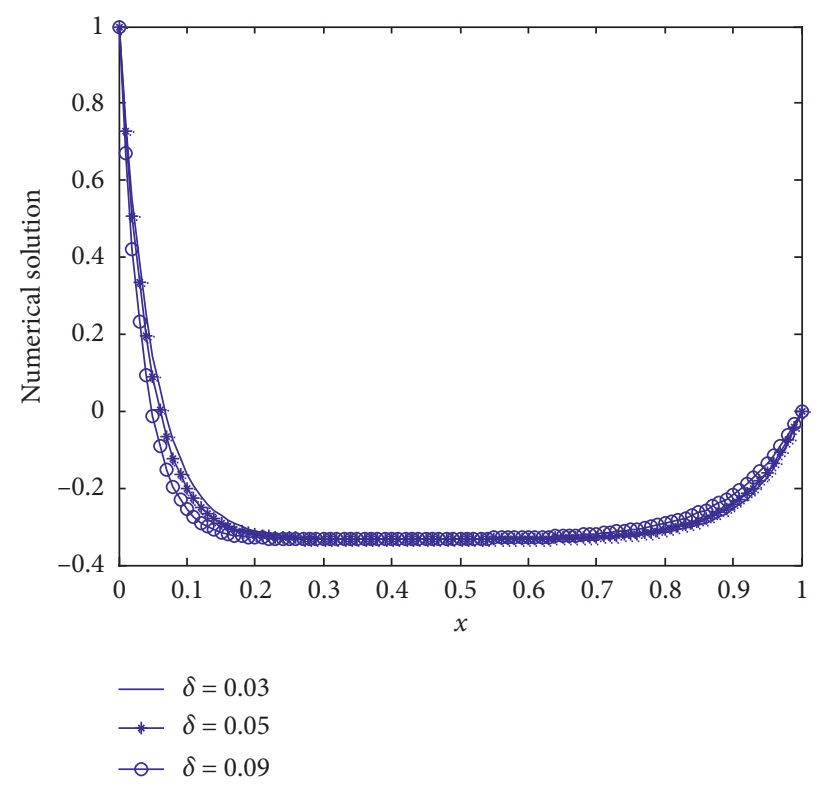

Figure 2: The numerical solution of Example 2 with $\varepsilon=0.01$ and $N=100$. 
TABle 5: The maximum absolute errors of Example 3 for different values of $\delta$ with $\varepsilon=0.1$.

\begin{tabular}{|c|c|c|c|c|c|}
\hline$\delta \downarrow$ & $N=100$ & $N=200$ & $N=300$ & $N=400$ & $N=500$ \\
\hline \multicolumn{6}{|c|}{ Present method } \\
\hline 0.03 & $4.0323 e-08$ & $2.5200 e-09$ & $4.9716 e-10$ & $1.5799 e-10$ & $6.3758 e-11$ \\
\hline 0.05 & $3.9610 e-08$ & $2.4762 e-09$ & $4.8871 e-10$ & $1.5567 e-10$ & $7.2701 e-11$ \\
\hline 0.09 & $3.8377 e-08$ & $2.3991 e-09$ & $4.7227 e-10$ & $1.5806 e-10$ & $6.1942 e-11$ \\
\hline \multicolumn{6}{|c|}{ Method in [17] } \\
\hline 0.03 & $2.5991 e-03$ & $1.2872 e-03$ & $8.5528 e-04$ & $6.4039 e-04$ & $5.1179 e-04$ \\
\hline 0.05 & $2.6270 e-03$ & $1.3013 e-03$ & $8.6474 e-04$ & $6.4750 e-04$ & $5.1749 e-04$ \\
\hline 0.09 & $2.6813 e-03$ & $1.3289 e-03$ & $8.8320 e-04$ & $6.6139 e-04$ & $5.2863 e-04$ \\
\hline
\end{tabular}

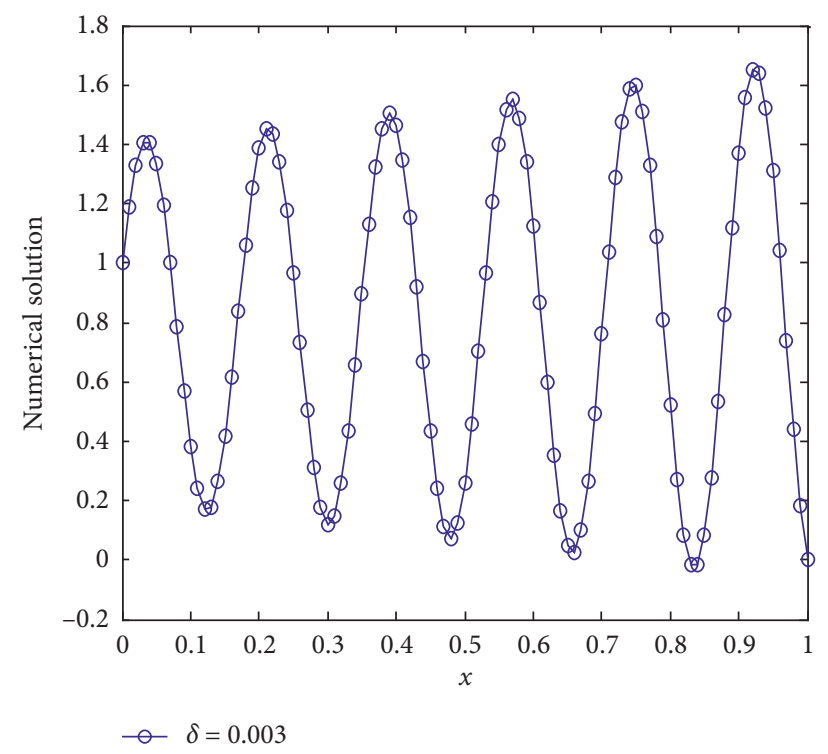

FIgURE 3: The numerical solution of Example 3 with $\varepsilon=0.001$ and $N=100$.

TABLE 6: The maximum absolute errors of Example 4 for different values of $\delta$ with $\varepsilon=0.1$.

\begin{tabular}{|c|c|c|c|c|c|}
\hline$\delta \downarrow$ & $N=100$ & $N=200$ & $N=300$ & $N=400$ & $N=500$ \\
\hline \multicolumn{6}{|c|}{ Present method } \\
\hline 0.03 & $1.5160 e-07$ & $9.4743 e-09$ & $1.8715 e-09$ & $5.9186 e-10$ & $2.4306 e-10$ \\
\hline 0.05 & $1.5697 e-07$ & $9.8097 e-09$ & $1.9379 e-09$ & $6.1294 e-10$ & $2.5132 e-10$ \\
\hline 0.09 & $1.7120 e-07$ & $1.0702 e-08$ & $2.1140 e-09$ & $6.6867 e-10$ & $2.7376 e-10$ \\
\hline \multicolumn{6}{|c|}{ Method in [17] } \\
\hline 0.03 & $1.5929 e-02$ & $7.4850 e-03$ & $4.8816 e-03$ & $3.6202 e-03$ & $2.8764 e-03$ \\
\hline 0.05 & $1.5470 e-02$ & $7.2782 e-03$ & $4.7473 e-03$ & $3.5209 e-03$ & $2.7975 e-03$ \\
\hline 0.09 & $2.1396 e-02$ & $1.0097 e-02$ & $6.5922 e-03$ & $4.8916 e-03$ & $3.8879 e-03$ \\
\hline
\end{tabular}

The graph of the computed solution for $\varepsilon=0.001$ and $\delta=0.003$ is also given in Figure 3 .

Example 4. Consider the singularly perturbed delay reaction-diffusion equation with oscillatory behavior

$$
\varepsilon y^{\prime \prime}(x)+y(x-\delta)+2 y(x)=1,
$$

under the interval and boundary conditions

$$
\begin{gathered}
y(x)=1, \\
-\delta \leq x \leq 0, \\
y(1)=0 .
\end{gathered}
$$

The maximum absolute errors are presented in Table 6 for different values of $\delta$.

The graph of the computed solution for $\varepsilon=0.001$ and $\delta=0.003$ is also given in Figure 4 . 


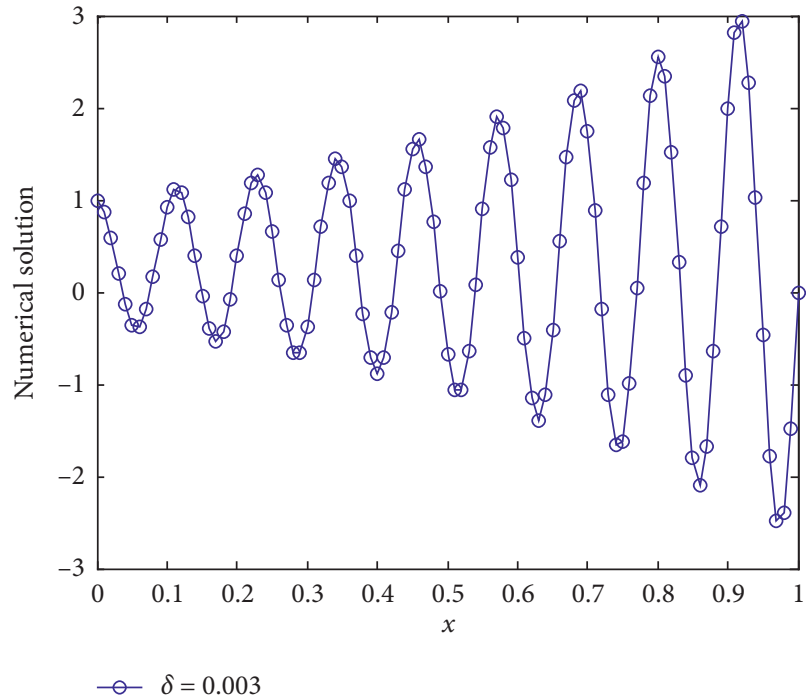

Figure 4: The numerical solution of Example 4 with $\varepsilon=0.001$ and $N=100$.

TABle 7: Rate of convergence $\rho$ for $\varepsilon=0.1$ and $\delta=0.05$.

\begin{tabular}{lccccc}
\hline$h$ & $h / 2$ & $Z_{h}$ & $h / 4$ & $Z_{h / 2}$ & $\rho$ \\
\hline Example 1 & & & & & \\
$1 / 100$ & $1 / 200$ & $1.1796 e-09$ & $1 / 400$ & $7.3727 e-11$ & 4.0000 \\
$1 / 200$ & $1 / 400$ & $7.3727 e-11$ & $1 / 800$ & $4.8657 e-12$ & 3.9215 \\
$1 / 300$ & $1 / 600$ & $1.4279 e-11$ & $1 / 1200$ & $9.1621 e-13$ & 3.9621 \\
Example 2 & & & & & \\
$1 / 100$ & $1 / 200$ & $7.7093 e-09$ & $1 / 400$ & $5.1758 e-10$ & 3.8967 \\
$1 / 200$ & $1 / 400$ & $5.1758 e-10$ & $1 / 800$ & $3.0120 e-11$ & 4.1030 \\
$1 / 300$ & $1 / 600$ & $9.5110 e-11$ & $1 / 1200$ & $6.0554 e-12$ & 3.9733 \\
\hline
\end{tabular}

TABLE 8: Rate of convergence $\rho$ for $\varepsilon=0.1$ and $\delta=0.03$.

\begin{tabular}{lccccc}
\hline$h$ & $h / 2$ & $Z_{h}$ & $h / 4$ & $Z_{h / 2}$ & $\rho$ \\
\hline Example 3 & & & & & \\
$1 / 100$ & $1 / 200$ & $4.0323 e-08$ & $1 / 400$ & $2.5200 e-09$ & 4.0001 \\
$1 / 200$ & $1 / 400$ & $2.5200 e-09$ & $1 / 800$ & $1.5799 e-10$ & 3.9955 \\
$1 / 300$ & $1 / 600$ & $4.9716 e-10$ & $1 / 1200$ & $3.0522 e-11$ & 4.0258 \\
Example 4 & & & & & \\
$1 / 100$ & $1 / 200$ & $1.5160 e-07$ & $1 / 400$ & $9.4743 e-09$ & 4.0001 \\
$1 / 200$ & $1 / 400$ & $9.4743 e-09$ & $1 / 800$ & $5.9186 e-10$ & 4.0007 \\
$1 / 300$ & $1 / 600$ & $1.8715 e-09$ & $1 / 1200$ & $1.1710 e-10$ & 3.9984 \\
\hline
\end{tabular}

The rate of convergence $(\rho)$ : in the same way, in equation (40), one can define $z_{h / 2}$ by replacing $h$ by $h / 2$ and $N-1$ by $2 N-1$, that is,

$$
z_{h / 2}=\max _{i}\left|y_{i}^{h / 2}-y_{i}^{h / 4}\right|, \quad \text { for } i=1,2, \ldots, 2 N-1 .
$$

The computational rate of convergence $\rho$ is also obtained by using double mesh principle defined as follows [9]:

$$
\rho=\frac{\left(\log \left(z_{h}\right)-\log \left(z_{h / 2}\right)\right)}{\log 2} .
$$

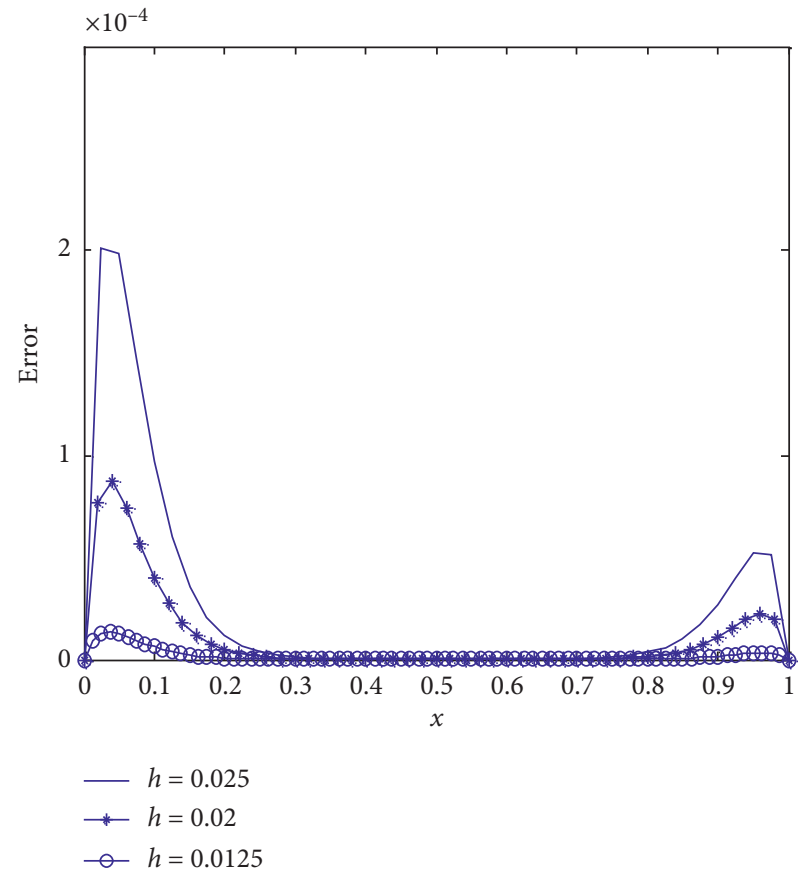

FIgURE 5: The point-wise absolute errors of Example 2 for different values of mesh size $h, \varepsilon=2^{-8}$, and $\delta=0.5 \varepsilon$.

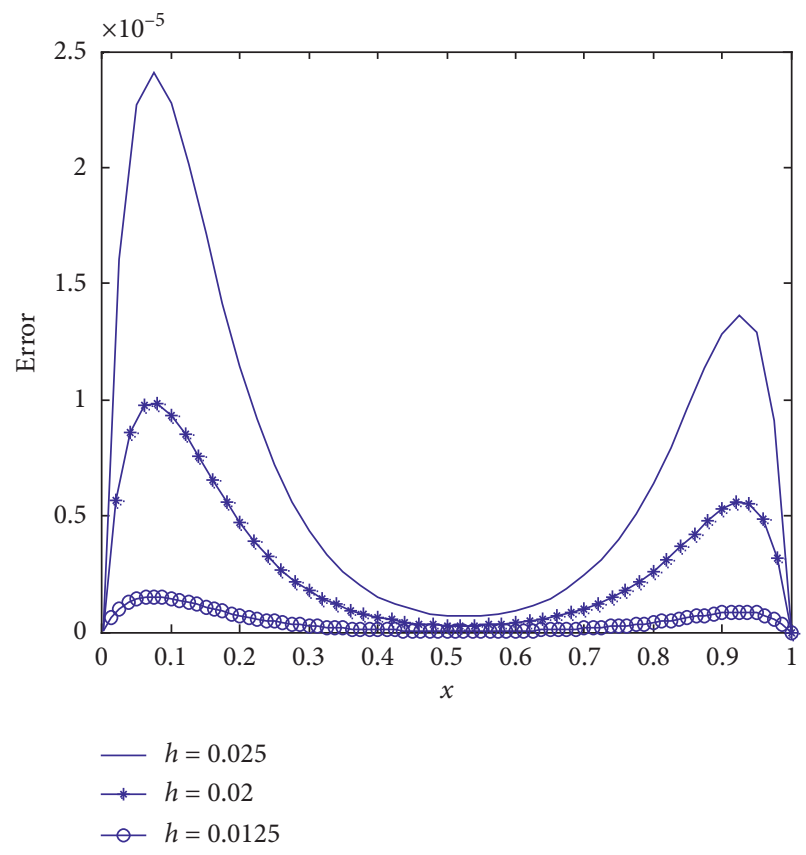

FIGURE 6: The point-wise absolute errors of Example 1 for different values of mesh size $h, \varepsilon=2^{-8}$, and $\delta=0.5 \varepsilon$.

The following tables (Tables 7 and 8) show the rate of convergence $\rho$ of the present method for different values of the mesh size $h$. 


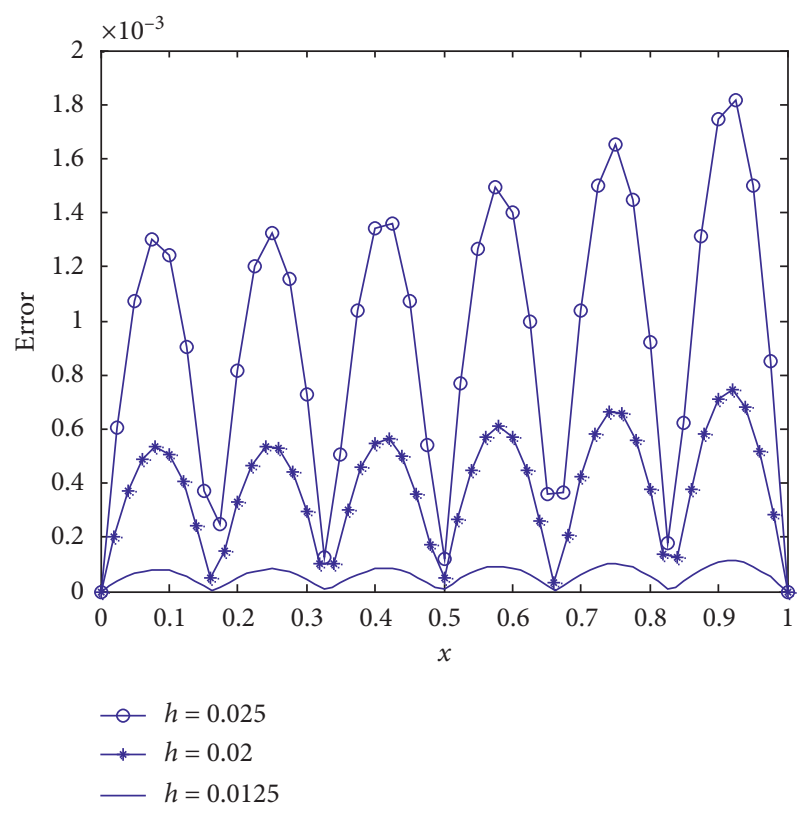

Figure 7: The point-wise absolute errors of Example 3 for different values of mesh size $h, \varepsilon=2^{-8}$, and $\delta=0.5 \varepsilon$.

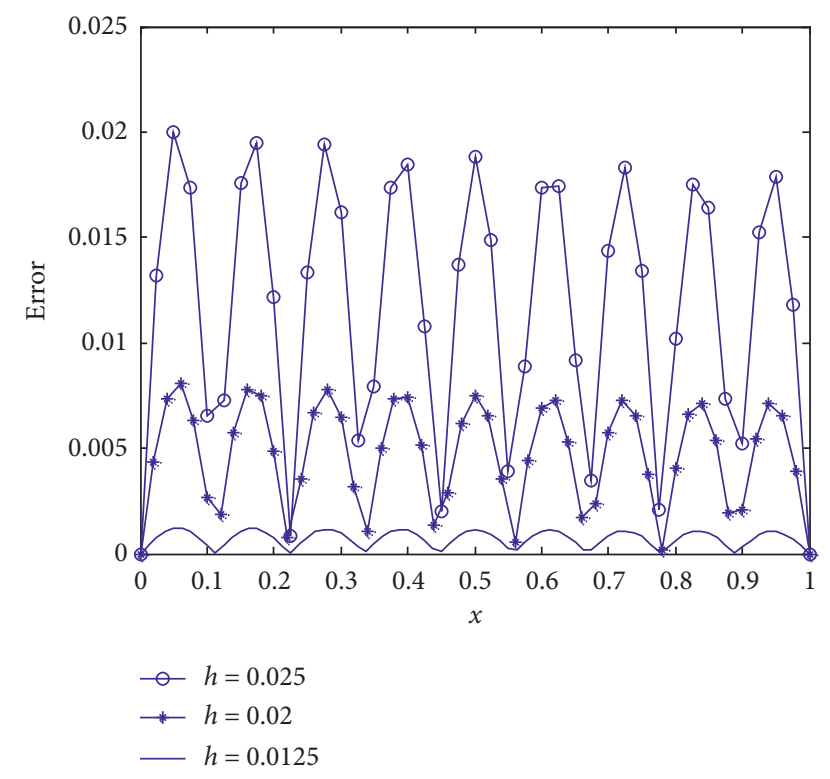

FIGURE 8: The point-wise absolute errors of Example 4 for different values of mesh size $h, \varepsilon=2^{-8}$, and $\delta=0.5 \varepsilon$.

4.1. The Effect of Delay Term on the Solution Profile. To analyze the effect of the delay term on the solution profile of the problem, the numerical solution of the problem for different values of the delay parameters has been given by the following graphs.

\section{Discussion and Conclusion}

Fourth-order fitted operator numerical method for solving singularly perturbed reaction-diffusion with delay has been presented. To demonstrate the efficiency of the method, four model examples without exact solutions have been considered for different values of the perturbation parameter $\varepsilon$ and delay parameter $\delta$, and also results are presented in the tables and figures. It is observed that, from the tables, the present method approximates the solution and the stability and convergence of the method is established well. The effect of the delay on the solution of singularly perturbed delay reaction-diffusion equation is showed by plotting graphs of four model examples. Two model examples of twin layers behavior and two model examples with oscillatory layers have been considered and solved for different values of perturbation parameter $\varepsilon$, delay parameter $\delta$, and mesh size $h$. The numerical solutions are tabulated (Tables 1-6) in terms of maximum absolute errors and observed that the present method improves the findings in [17]. Also, it is significant that all of the maximum absolute errors decrease rapidly as $N$ increases. The results presented in Tables 7 and 8 confirmed that computational rate of convergence as well as the theoretical estimate indicates that the method is a fourthorder convergent.

Furthermore, to investigate the effect of delay on the solution of the problem, numerical solutions have been presented using graphs. Accordingly, when the order of the coefficient of the delay term is of $O(1)$, the delay affects the boundary layer solution but maintains the layer behavior (Figure 1). When the delay parameter is of $O(\varepsilon)$, the solution maintains layer behavior although the coefficient of the delay term in the equation of $O(1)$ and the delay increases, the thickness of the left boundary layer decreases while that of the right boundary layer increases (Figure 2). For the oscillatory behavior case, one can conclude that the solution oscillates throughout the domain for different values of delay parameter $\delta$ (Figures 3 and 5). In a concise manner, the present method gives more accurate solution and is uniformly convergent for solving singularly perturbed delay reaction-diffusion equations with twin layer and oscillatory behavior. Also it can see that, as mesh size $h$ decreases, the absolute errors also decrease from Figures 5-8.

\section{Data Availability}

No data were used to support the study.

\section{Conflicts of Interest}

The authors declare that they have no conflicts of interest.

\section{Authors' Contributions}

HG proposed the main idea of this paper. $\mathrm{HG}, \mathrm{SB}$, and $\mathrm{AD}$ prepared the manuscript and performed all the steps of the proofs in this research. All authors contributed equally and significantly in writing this paper. All authors read and approved the final manuscript.

\section{Acknowledgments}

The authors wish to express their thanks to Jimma University, College of Natural Sciences, for technical support 
and the authors of literatures for the provided scientific aspects and idea for this work.

\section{References}

[1] D. K. Swamy, K. Phaneendra, and Y. N. Reddy, "Accurate numerical method for singularly Perturbed differential-difference equations with mixed shifts," Khayyam Journal of Mathematics, vol. 4, no. 1, pp. 110-122, 2018.

[2] A. Longtin and J. G. Milton, "Complex oscillations in the human pupil light reflex with mixed and delayed feedback," Mathematical Bio Sciences, vol. 90, no. 1-2, pp. 183-199, 1988.

[3] M. W. Derstine, H. M. Gibbs, F. A. Hopf, and D. L. Kaplan, "Bifurcation gap in a hybrid optically bistable system," Physical Review A, vol. 26, no. 6, pp. 3720-3722, 1982.

[4] V. Glizer, "Asymptotic analysis and solution of a finite-horizon ho control problem for singularly-perturbed linear systems with small state delay," Journal of Optimization Theory and Applications, vol. 117, pp. 295-325, 2003.

[5] G. B. Soujanya and Y. N. Reddy, "Computational method for singularly perturbed delay differential equations with layer or oscillatory behaviour," Applied Mathematics \& Information Sciences, vol. 10, no. 2, pp. 527-536, 2016.

[6] K. Phaneendra, G. B. S. L. Soujanya, and Y. N. Reddy, "Numerical solution of second order singularly perturbed differential-difference equation with negative shift," International Journal of Nonlinear Science, vol. 18, no. 3, pp. 200-209, 2014.

[7] P. P. Chakravarthy, S. D. Kumar, and R. N. Rao, “An exponentially fitted finite difference scheme for a class of singularly perturbed delay differential equations with large delays," Ain Shams Engineering Journal, vol. 8, no. 4, pp. 663-671, 2017.

[8] L. Sirisha and Y. N. Reddy, "Numerical solution of singularly perturbed differential-difference equations with dual layer," American Journal of Applied Mathematics and Statistics, vol. 2, no. 5, pp. 336-343, 2014.

[9] E. R. Doolan, J. J. H. Miller, and W. H. A. Schilders, Uniform Numerical Methods for Problems with Initial and Boundary Layers, Boole Press, Dublin, Ireland, 1980.

[10] M. K. Kadalbajoo and K. K. Sharma, "Numerical analysis of singularly perturbed delay differential equations with layer behavior," Applied Mathematics and Computation, vol. 157, no. 1, pp. 11-28, 2004.

[11] H. G. Roos, M. Stynes, and L. Tobiska, Numerical Methods for Singularly Perturbed Differential Equations, Springer, Berlin, Germany, 1996.

[12] F. Gemechis, G. Gashu, A. Tesfaye, and Y. N. Reddy, "Numerical solution of singularly perturbed delay reaction-diffusion equations with layer or oscillatory behavior," American Journal of Numerical Analysis, vol. 5, no. 1, pp. 1-10, 2017.

[13] R. E. OMalley, Singular Perturbation Methods for Ordinary Differential Equations, Springer-Verlag, New York, NY, USA, 1991.

[14] E. O’Riordan and M. Stynes, “A globally uniformly convergent finite element method for a singularly perturbed elliptic problem in two dimensions," Mathematics of Computation, vol. 57, no. 195, pp. 47-62, 1991.

[15] C. Clavero, J. L. Gracia, and J. C. Jorge, "High-order numerical methods for one-dimensional parabolic singularly perturbed problems with regular layers," Numerical Methods for Partial Differential Equations, vol. 21, no. 1, pp. 149-169, 2005.

[16] M. M. Woldaregay and G. F. Duressa, "Parameter uniform numerical method for singularly perturbed differential difference equations," Journal of the Nigerian Mathematical Society, vol. 38, no. 2, pp. 223-245, 2009.

[17] D. K. Swamy, K. Phaneendra, A. B. Babu, and Y. N. Reddy, "Computational method for singularly perturbed delay differential equations with twin layers or oscillatory behavior," Ain Shams Engineering Journal, vol. 6, no. 1, pp. 391-398, 2015. 